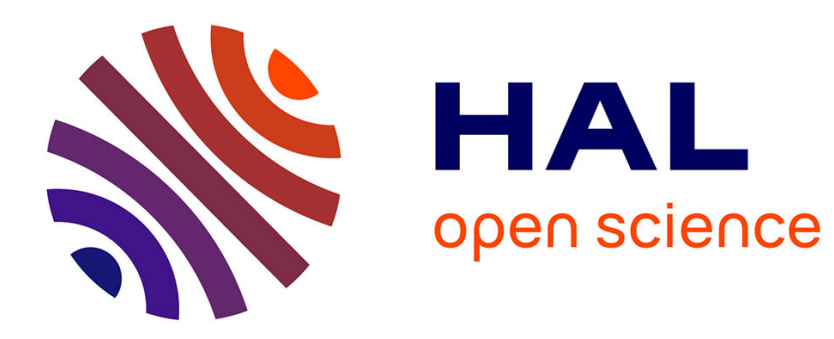

\title{
La population des continents et des états en 2014
}

Jean-Paul Sardon

\section{To cite this version:}

Jean-Paul Sardon. La population des continents et des états en 2014. Population\&Avenir, 2014. hal-02543949

\section{HAL Id: hal-02543949 \\ https://hal.science/hal-02543949}

Submitted on 22 Apr 2020

HAL is a multi-disciplinary open access archive for the deposit and dissemination of scientific research documents, whether they are published or not. The documents may come from teaching and research institutions in France or abroad, or from public or private research centers.
L'archive ouverte pluridisciplinaire HAL, est destinée au dépôt et à la diffusion de documents scientifiques de niveau recherche, publiés ou non, émanant des établissements d'enseignement et de recherche français ou étrangers, des laboratoires publics ou privés. 


\section{La population des continents et des États en 2014}

\author{
Population \& Avenir présente et analyse \\ les statistiques mondiales de popula- \\ tion, par continents et sous-continents, \\ de tous les États membres de l'Organi- \\ sation des Nations Unies (ONU) et de \\ toutes les entités géographiques dont la \\ population atteint ou dépasse 150000 \\ habitants au moins. Ces données, ras- \\ semblées annuellement par le Population \\ Reference Bureau ${ }^{1}$, sont ici complétées \\ par le seuil de remplacement ${ }^{2}$.
}

\section{Croissance démographique mondiale : l'Inde, pour près d'un cinquième}

Ces tableaux présentent peu de différences par rapport aux précédents du fait de l'inertie des phénomènes démographiques. La population dans le monde aurait progressé de 111 millions d'habitants entre les $1^{\text {er }}$ juillet 2013 et 2014. Comme au cours des années précédentes, la croissance la plus forte, en légère augmentation, proviendrait de l'Inde (+19,7 millions). Cette année encore la croissance chinoise (+6,7 millions) ne figure qu'au quatrième rang, précédée

1. Organisme indépendant situé à Washington, USA.

2. Recalculé pour prendre en compte la dernière révision (2012) des projections des Nations-Unies. Nous remercions Pavlos Baltas pour son travail annuel de conversion des données.

\section{Le contenu des 15 colonnes}

Les données ci-après concernent :

1. La superficie (en milliers de $\mathrm{km}^{2}$ ), sans les eaux intérieures

2. La population estimée au 1er juillet 2014 (en millions d'habitants) ;

3. La projection moyenne de population pour 2050 (en millions d'habitants), fondée sur les hypothèses moyennes des Nations unies quant à l'évolution de la fécondité, de la mortalité et de la migration ;

4. Le taux de natalité (pour mille habitants); 5. Le taux de mortalité (pour mille habitants) ;

6. Le taux d'accroissement nature ${ }^{1}$ (pour cent habitants) :

7. Le taux d'accroissement migratoire (pour mille habitants)

1. Des arrondis peuvent expliquer que ce taux diffère légèrement de la différence entre taux de natalité et taux de mortalité.
8. Le taux de mortalité infantile (pour mille naissances) ;

9. Lindice de fécondité (nombre moyen d'enfants auxquels une femme donnerait naissance, si les taux actuels de fécondité par âge demeuraient constants pendant toute sa vie reproductive) ;

10. L'indice de fécondité nécessaire pour assurer le simple remplacement des générations (calculé par Population \& Avenir à partir des estimations des Nations unies pour la période 2010-2015) ;

11. Le pourcentage de la population de moins de 15 ans :

12. Le pourcentage de la population de 65 ans ou plus :

13. L'espérance de vie à la naissance du sexe masculin ;

14. L'espérance de vie à la naissance du sexe féminin :

15. Le produit national brut (PNB) par habitant en parité de pouvoir d'achat (PPA), en dollars US pour l'an 2013 également par celle du Brésil ( $+7,3$ millions) et de l'Éthiopie (6,7 millions). Viennent ensuite le Soudan $(+4,6)$, le Nigeria et les Philippines $(+3,9)$, le Pakistan $(+3,3)$, l'Égypte $(+3,2)$ et l'Indonésie $(+3,0)$.

Ainsi, alors que parmi les vingt pays les plus peuplés ne figurent que cinq pays africains, neuf d'entre eux apparaissent dans le classement des croissances absolues les plus fortes ${ }^{3}$.

À l'opposé, les estimations des populations de l'Ukraine, de la Roumanie et du Kenya sont en diminution, respectivement, de 2,6, 1,3 et 1,0 millions.

À l'échelle des continents, l'Asie continue de concentrer la part la plus élevée de la croissance de la population mondiale ( $49 \%$ contre $62 \%$ il y a deux ans). Mais c'est en Afrique, et particulièrement en Afrique orientale, que la croissance relative annuelle est la plus élevée (3,3\% et 4,4 \% respectivement, contre $1,1 \%$ en Asie).

\section{Des écarts considérables de fécondité}

Pour l'ensemble de la planète, l'indice de fécondité 2014 reste stable avec 2,5 enfants par femme.

Toutefois, ce chiffre cache une grande diversité de situations. La fécondité serait en baisse notable dans quelques pays, notamment de 1,0 enfant par femme au Liberia, de 0,7 au Rwanda, en Éthiopie et en Mauritanie. Mais elle serait en hausse au Sud Soudan $(1,9)$, au Soudan $(0,6)$ et en Égypte $(0,5)$.

Dans tous les continents et grandes régions, la fécondité est stabilisée ou en baisse, sauf en Afrique septentrionale où elle augmenterait de 0,2 enfant par femme sous l'effet de son évolution au Soudan et en Égypte. C'est également la situation dans les pays les plus peuplés. Parmi les vingt premiers, seuls l'Égypte (15), la République Démocratique du Congo $\left(19^{\mathrm{e}}\right)$ et la Thaillande $\left(20^{\mathrm{e}}\right)$ enregistrent une élévation de leur fécondité.

La fécondité de l'Union européenne ${ }^{4}$ se réduit légèrement de 1,6 à 1,5, creusant un peu plus l'écart avec les États-Unis $(1,9)$.

Dans 38 pays, dont 29 d'Europe, l'indicateur ne dépasserait pas 1,5 enfant par femme. Toutefois, les niveaux les plus faibles n'atteignent plus des valeurs aussi basses qu'en 2012 . Seuls deux pays, Andorre et la Bosnie-Herzégovine, enregistrent une valeur de 1,2 enfant par femme.

Dans l'ensemble de la planète, $\mathbf{8 8}$ pays, peuplés de 3425 millions d'habitants (soit 47,3\% du total mondial), ne remplacent pas leurs générations. A l'opposé, 51 pays (dont 40 d'Afrique), peuplés de 985 millions d'habitants (13,6\% du total mondial) ont des fécondités dépassant 4,0 enfants par femme $e^{5}$. A l'échelle continentale, seule l'Afrique assure son remplacement.

3. Pour plus de détail sur lévolution de la population en Afrique voir : « Afrique : que penser de sa fécondité élevée ? », Population \& Avenir, n 719, septembre-octobre 2014. 4. Cf. également Dumont, Gérard-François, Verluise, Pierre, Géopolitique de l'Europe, Paris, Armand Colin - Sedes, 2014.

5. May, John, « Monde : les nouveaux défis démographiques », Population \& Avenir, $n^{\circ} 78$, mai-juin 2014 


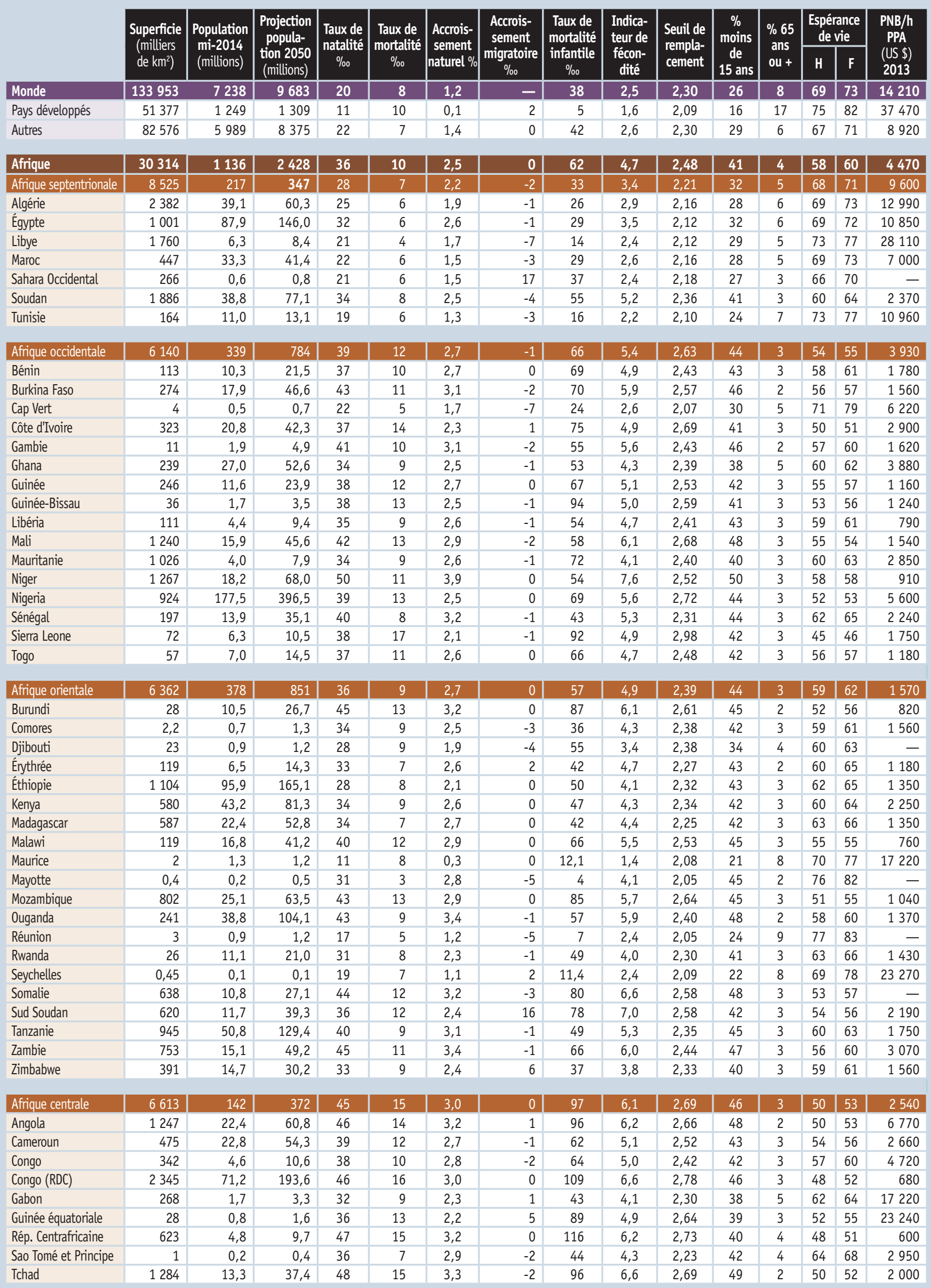


DISS POPUIATHIONS

\begin{tabular}{|c|c|c|c|c|c|c|c|c|c|c|c|c|c|c|c|}
\hline & \multirow{2}{*}{$\begin{array}{c}\text { Superficie } \\
(\text { milliers } \\
\left.\text { de } \mathrm{km}^{2}\right)\end{array}$} & \multirow{2}{*}{$\begin{array}{c}\text { Population } \\
\text { mi-2014 } \\
\text { (millions) }\end{array}$} & \multirow{2}{*}{$\begin{array}{c}\text { Projection } \\
\text { popula- } \\
\text { tion } 2050 \\
\text { (millions) }\end{array}$} & \multirow{2}{*}{$\begin{array}{c}\text { Taux de } \\
\text { natalité } \\
\% 0\end{array}$} & \multirow{2}{*}{$\begin{array}{c}\text { Taux de } \\
\text { mortalité } \\
\% 0\end{array}$} & \multirow{2}{*}{$\begin{array}{c}\text { Accrois- } \\
\text { sement } \\
\text { naturel \% }\end{array}$} & \multirow{2}{*}{$\begin{array}{c}\text { Accrois- } \\
\text { sement } \\
\text { migratoire } \\
\% 00\end{array}$} & \multirow{2}{*}{$\begin{array}{c}\text { Taux de } \\
\text { mortalité } \\
\text { infantile } \\
\%\end{array}$} & \multirow{2}{*}{$\begin{array}{l}\text { Indica- } \\
\text { teur de } \\
\text { fécon- } \\
\text { dité }\end{array}$} & \multirow{2}{*}{$\begin{array}{l}\text { Seuil de } \\
\text { rempla- } \\
\text { cement }\end{array}$} & \multirow{2}{*}{\begin{tabular}{c|c}
$\%$ \\
moins \\
de \\
15 ans
\end{tabular}} & \multirow{2}{*}{$\begin{array}{c}\% 65 \\
\text { ans } \\
\text { ou + }\end{array}$} & \multicolumn{2}{|c|}{$\begin{array}{c}\text { Espérance } \\
\text { de vie }\end{array}$} & \multirow{2}{*}{$\begin{array}{c}\text { PNB/h } \\
\text { PPA } \\
\text { (US \$) } \\
2013\end{array}$} \\
\hline & & & & & & & & & & & & & H & F & \\
\hline Afrique Australe & 2674 & 61 & 75 & 21 & 11 & 1,0 & 3 & 44 & 2,4 & 2,32 & 30 & 5 & 57 & 60 & 11840 \\
\hline Afrique du Sud & 1221 & 53,7 & 64,1 & 20 & 11 & 1,0 & 3 & 42 & 2,3 & 2,31 & 29 & 5 & 58 & 61 & 12240 \\
\hline Botswana & 582 & 2,0 & 2,8 & 24 & 17 & 0,7 & 2 & 32 & 2,6 & 2,49 & 34 & 4 & 48 & 47 & 15500 \\
\hline Lesotho & 30 & 1,9 & 2,7 & 30 & 21 & 0,9 & -6 & 82 & 3,3 & 2,51 & 36 & 6 & 42 & 45 & 3320 \\
\hline Namibie & 824 & 2,3 & 3,7 & 30 & 7 & 2,2 & 0 & 39 & 3,6 & 2,19 & 36 & 4 & 62 & 67 & 9590 \\
\hline Swaziland & 17 & 1,3 & 1,8 & 30 & 14 & 1,6 & -1 & 65 & 3,4 & 2,60 & 38 & 3 & 50 & 49 & 6220 \\
\hline
\end{tabular}

\begin{tabular}{|l|r|r|r|r|r|r|r|r|r|r|r|r|r|r|r|}
\hline Amérique & $\mathbf{4 0} 135$ & $\mathbf{9 7 2}$ & $\mathbf{1 2 1 7}$ & $\mathbf{1 6}$ & $\mathbf{7}$ & $\mathbf{0 , 9}$ & $\mathbf{0}$ & $\mathbf{1 5}$ & $\mathbf{2 , 1}$ & $\mathbf{2 , 1 0}$ & $\mathbf{2 4}$ & $\mathbf{1 0}$ & $\mathbf{7 3}$ & $\mathbf{7 9}$ & $\mathbf{2 7} 4 \mathbf{4 2 0}$ \\
\hline Amérique du Nord & 19600 & 353 & 444 & 12 & 8 & 0,4 & 3 & 5 & 1,8 & 2,08 & 19 & 14 & 77 & 81 & 52810 \\
\hline Canada & 9971 & 35,5 & 48,4 & 11 & 7 & 0,4 & 8 & 4,8 & 1,6 & 2,08 & 16 & 15 & 79 & 84 & 42590 \\
\hline Etats-Unis & 9629 & 317,7 & 395,3 & 13 & 8 & 0,4 & 3 & 5,4 & 1,9 & 2,08 & 19 & 14 & 76 & 81 & 53960 \\
\hline
\end{tabular}

\begin{tabular}{|c|c|c|c|c|c|c|c|c|c|c|c|c|c|c|c|}
\hline Amérique Latine & 20535 & 618 & 773 & 18 & 6 & 1,2 & -1 & 18 & 2,2 & 2,12 & 27 & 7 & 71 & 78 & 12900 \\
\hline Amérique centrale & 2480 & 165 & 222 & 21 & 6 & 1,5 & -2 & 16 & 2,4 & 2,11 & 30 & 6 & 71 & 77 & 13880 \\
\hline Belize & 23 & 0,4 & 0,6 & 23 & 4 & 1,9 & 6 & 14 & 2,6 & 2,10 & 36 & 4 & 71 & 77 & 8160 \\
\hline Costa Rica & 51 & 4,8 & 6,1 & 15 & 4 & 1,1 & 2 & 8,7 & 1,8 & 2,08 & 24 & 7 & 77 & 82 & 13570 \\
\hline El Salvador & 21 & 6,4 & 6,9 & 20 & 7 & 1,4 & -7 & 17 & 2,2 & 2,12 & 30 & 7 & 68 & 77 & 7490 \\
\hline Guatemala & 109 & 15,9 & 31,3 & 31 & 5 & 2,6 & -1 & 23 & 3,8 & 2,15 & 40 & 5 & 68 & 76 & 7130 \\
\hline Honduras & 112 & 8,2 & 11,7 & 24 & 5 & 2,0 & -1 & 24 & 2,8 & 2,16 & 35 & 4 & 71 & 76 & 4270 \\
\hline Mexique & 1958 & 119,7 & 150,8 & 19 & 6 & 1,4 & -2 & 13 & 2,2 & 2,09 & 28 & 6 & 71 & 77 & 16110 \\
\hline Nicaragua & 130 & 6,2 & 8,4 & 23 & 5 & 1,9 & -6 & 17 & 2,5 & 2,12 & 33 & 5 & 72 & 78 & 4440 \\
\hline Panama & 76 & 3,9 & 5,6 & 20 & 5 & 1,5 & 2 & 14 & 2,5 & 2,11 & 28 & 7 & 74 & 79 & 19290 \\
\hline Caraïbes & 234 & 43 & 49 & 18 & 8 & 1,1 & -4 & 33 & 2,3 & 2,19 & 26 & 9 & 70 & 75 & 11740 \\
\hline Antigua et Barbuda & 0,4 & 0,1 & 0,1 & 14 & 5 & 0,8 & 5 & 16 & 1,8 & 2,07 & 24 & 7 & 74 & 80 & 20070 \\
\hline Bahamas & 14 & 0,4 & 0,5 & 16 & 5 & 1,1 & 2 & 17 & 2,0 & 2,12 & 26 & 6 & 72 & 77 & 21540 \\
\hline Barbade & 0,4 & 0,3 & 0,3 & 12 & 8 & 0,4 & 1 & 10 & 1,8 & 2,08 & 20 & 13 & 73 & 78 & 15080 \\
\hline Cuba & 111 & 11,2 & 9,3 & 11 & 8 & 0,3 & -4 & 4,6 & 1,7 & 2,08 & 17 & 13 & 76 & 80 & 18520 \\
\hline Curacao & 0,4 & 0,2 & 0,2 & 13 & 8 & 0,5 & 4 & 9,8 & 2,2 & 2,12 & 19 & 15 & 74 & 81 & - \\
\hline Dominique & 0,8 & 0,1 & 0,1 & 13 & 8 & 0,5 & -6 & 21 & 2,0 & 2,09 & 22 & 10 & 71 & 76 & 9800 \\
\hline Grenade & 0,3 & 0,1 & 0,1 & 16 & 7 & 0,8 & -2 & 9 & 2,0 & - & 27 & 7 & 67 & 73 & 11120 \\
\hline Guadeloupe & 1,7 & 0,4 & 0,4 & 13 & 7 & 0,6 & -7 & 8,1 & 2,2 & 2,10 & 21 & 15 & 77 & 84 & - \\
\hline Haïti & 28 & 10,8 & 16,8 & 28 & 9 & 1,9 & -3 & 59 & 3,4 & 2,07 & 35 & 4 & 61 & 65 & 1710 \\
\hline Jamaïque & 11 & 2,7 & 2,7 & 18 & 7 & 1,1 & -5 & 21 & 2,3 & 2,34 & 27 & 8 & 71 & 76 & 8480 \\
\hline Martinique & 1,1 & 0,4 & 0,4 & 12 & 7 & 0,4 & -10 & 8 & 1,9 & 2,13 & 19 & 17 & 79 & 85 & - \\
\hline Porto Rico & 9 & 3,6 & 3 & 11 & 8 & 0,3 & -13 & 8,1 & 1,6 & 2,07 & 19 & 16 & 75 & 83 & 22730 \\
\hline Rép. dominicaine & 49 & 10,4 & 13,2 & 21 & 6 & 1,5 & -3 & 25 & 2,5 & 2,08 & 31 & 6 & 70 & 76 & 11150 \\
\hline $\begin{array}{l}\text { St Christophe et Nié- } \\
\text { vès }\end{array}$ & 0,3 & 0,1 & 0,1 & 13 & 7 & 0,6 & 7 & 17 & 1,6 & 2,13 & 22 & 8 & 73 & 78 & 20400 \\
\hline St Vincent/Grenadines & 0,4 & 0,1 & 0,1 & 17 & 8 & 0,9 & -9 & 20 & 2,2 & - & 25 & 7 & 70 & 74 & 10610 \\
\hline Ste Lucie & 0,5 & 0,2 & 0,2 & 13 & 8 & 0,6 & 0 & 20 & 1,5 & 2,09 & 23 & 9 & 72 & 77 & 10350 \\
\hline Trinité et Tobago & 5 & 1,3 & 1 & 13 & 10 & 0,3 & -3 & 24 & 1,6 & 2,09 & 20 & 9 & 66 & 74 & 26210 \\
\hline Amérique du Sud & 17821 & 410 & 503 & 17 & 6 & 1,1 & 0 & 18 & 2,1 & 2,11 & 26 & 8 & 72 & 78 & 12620 \\
\hline Argentine & 2780 & 42,7 & 59,4 & 19 & 7 & 1,1 & 0 & 11,7 & 2,3 & 2,08 & 25 & 10 & 73 & 80 & - \\
\hline Bolivie & 1099 & 10,3 & 15,8 & 26 & 7 & 1,9 & 0 & 39 & 3,2 & 2,24 & 35 & 5 & 65 & 69 & 5750 \\
\hline Brésil & 8515 & 202,8 & 226,3 & 15 & 6 & 0,9 & 0 & 20 & 1,8 & 2,11 & 24 & 7 & 71 & 79 & 14750 \\
\hline Chili & 756 & 17,7 & 20,2 & 14 & 6 & 0,9 & 0 & 7,7 & 1,9 & 2,06 & 22 & 10 & 76 & 82 & 21030 \\
\hline Colombie & 1139 & 47,7 & 61,3 & 19 & 6 & 1,3 & -2 & 18 & 2,3 & 2,11 & 28 & 7 & 72 & 79 & 11890 \\
\hline Équateur & 284 & 16,0 & 23,4 & 23 & 5 & 1,8 & 0 & 17 & 2,8 & 2,12 & 31 & 7 & 72 & 78 & 10310 \\
\hline Guyana & 215 & 0,7 & 0,8 & 20 & 7 & 1,3 & -15 & 29 & 2,5 & 2,18 & 36 & 3 & 64 & 69 & 6550 \\
\hline Guyane française & 90 & 0,3 & 0,6 & 27 & 3 & 2,4 & 2 & 10 & 3,5 & 2,09 & 34 & 5 & 76 & 83 & - \\
\hline Paraguay & 407 & 6,9 & 10,1 & 23 & 6 & 1,7 & -1 & 29 & 2,8 & 2,16 & 33 & 5 & 71 & 75 & 7640 \\
\hline Pérou & 1285 & 30,8 & 40,1 & 20 & 5 & 1,5 & -3 & 16 & 2,4 & 2,13 & 29 & 6 & 72 & 77 & 11360 \\
\hline Surinam & 164 & 0,6 & 0,7 & 20 & 7 & 1,3 & -2 & 20 & 2,6 & 2,15 & 28 & 6 & 69 & 74 & 15860 \\
\hline Uruguay & 175 & 3,4 & 3,6 & 14 & 9 & 0,5 & -2 & 9,3 & 2,0 & 2,09 & 22 & 14 & 74 & 81 & 18930 \\
\hline Venezuela & 912 & 30,2 & 40,5 & 20 & 5 & 1,5 & 0 & 14,4 & 2,4 & 2,11 & 29 & 6 & 72 & 78 & 17890 \\
\hline
\end{tabular}




\section{D) HS POPULAHIONS}

\begin{tabular}{|c|c|c|c|c|c|c|c|c|c|c|c|c|c|c|c|}
\hline & \multirow{2}{*}{$\begin{array}{c}\text { Superficie } \\
(\text { milliers } \\
\left.\text { de }^{2} m^{2}\right)\end{array}$} & \multirow{2}{*}{$\begin{array}{c}\text { Population } \\
\text { mi-2014 } \\
\text { (millions) }\end{array}$} & \multirow{2}{*}{$\begin{array}{c}\text { Projection } \\
\text { popula- } \\
\text { tion } 2050 \\
\text { (millions) }\end{array}$} & \multirow{2}{*}{$\begin{array}{c}\text { Taux de } \\
\text { natalité } \\
\% 0\end{array}$} & \multirow{2}{*}{$\begin{array}{c}\text { Taux de } \\
\text { mortalité } \\
\% 0\end{array}$} & \multirow{2}{*}{$\begin{array}{c}\text { Accrois- } \\
\text { sement } \\
\text { naturel \% }\end{array}$} & \multirow{2}{*}{$\begin{array}{c}\text { Accrois- } \\
\text { sement } \\
\text { migratoire } \\
\% \%\end{array}$} & \multirow{2}{*}{$\begin{array}{c}\text { Taux de } \\
\text { mortalité } \\
\text { infantile } \\
\% 0\end{array}$} & \multirow{2}{*}{$\begin{array}{l}\text { Indica- } \\
\text { teur de } \\
\text { fécon- } \\
\text { dité }\end{array}$} & \multirow{2}{*}{$\begin{array}{l}\text { Seuil de } \\
\text { rempla- } \\
\text { cement }\end{array}$} & \multirow{2}{*}{\begin{tabular}{c|}
$\%$ \\
moins \\
de \\
15 ans
\end{tabular}} & \multirow{2}{*}{$\begin{array}{c}\% 65 \\
\text { ans } \\
\text { ou + }\end{array}$} & $\begin{array}{c}\text { Espér } \\
\text { de }\end{array}$ & $\begin{array}{l}\text { ance } \\
\text { ie }\end{array}$ & $\begin{array}{c}\text { PNB/h } \\
\text { PPA }\end{array}$ \\
\hline & & & & & & & & & & & & & H & $\mathbf{F}$ & $\begin{array}{l}\text { (US \$) } \\
2013\end{array}$ \\
\hline Asie & 31880 & 4351 & 5252 & 18 & 7 & 1,1 & 0 & 34 & 2,2 & 2,25 & 25 & 7 & 69 & 73 & 10380 \\
\hline Asie occidentale & 4832 & 255 & 387 & 22 & 5 & 1,7 & 2 & 25 & 2,9 & 2,13 & 30 & 5 & 71 & 76 & 22920 \\
\hline Arabie Saoudite & 2150 & 30,8 & 42,4 & 22 & 4 & 1,8 & 2 & 16 & 2,9 & 2,08 & 30 & 3 & 73 & 75 & 53780 \\
\hline Arménie & 30 & 3,0 & 2,8 & 14 & 9 & 0,5 & -8 & 10 & 1,6 & 2,19 & 19 & 11 & 71 & 78 & 8140 \\
\hline Azerbaïdjan & 87 & 9,5 & 12,1 & 18 & 6 & 1,3 & 0 & 11 & 2,2 & 2,26 & 22 & 6 & 71 & 77 & 16180 \\
\hline Bahrein & 0,7 & 1,3 & 1,8 & 15 & 2 & 1,3 & 20 & 8 & 2,1 & 2,07 & 21 & 2 & 75 & 78 & 36140 \\
\hline Chypre & 9 & 1,2 & 1,4 & 13 & 7 & 0,6 & -2 & 6 & 1,5 & 2,08 & 17 & 12 & 77 & 81 & 29570 \\
\hline Émirats Arabes Unis & 84 & 9,4 & 15,5 & 15 & 1 & 1,4 & 22 & 6 & 1,8 & 2,07 & 16 & 0 & 76 & 78 & 58090 \\
\hline Géorgie & 70 & 4,8 & 4,6 & 13 & 11 & 0,2 & -1 & 11 & 1,7 & 2,16 & 17 & 14 & 70 & 79 & 7040 \\
\hline Irak & 438 & 35,1 & 80,5 & 31 & 5 & 2,6 & 1 & 29 & 4,1 & 2,16 & 40 & 3 & 66 & 73 & 15220 \\
\hline Israël & 22 & 8,2 & 13,9 & 21 & 5 & 1,6 & 3 & 3 & 3,0 & 2,07 & 28 & 10 & 80 & 84 & 32140 \\
\hline Jordanie & 89 & 7,6 & 13,1 & 28 & 4 & 2,4 & -8 & 17 & 3,5 & 2,12 & 34 & 4 & 72 & 74 & 11660 \\
\hline Koweït & 18 & 3,7 & 6,6 & 18 & 2 & 1,7 & 35 & 8 & 2,4 & 2,08 & 23 & 2 & 73 & 75 & 88170 \\
\hline Liban & 10 & 5,0 & 5,3 & 13 & 4 & 0,9 & 20 & 9 & 1,5 & 2,08 & 20 & 9 & 78 & 82 & 17390 \\
\hline Oman & 310 & 4,1 & 6,3 & 20 & 2 & 1,8 & 39 & 9 & 2,8 & 2,09 & 22 & 3 & 75 & 78 & 52170 \\
\hline Palestiniens (Territ.) & 6 & 4,4 & 9,1 & 31 & 4 & 2,7 & -1 & 20 & 4,1 & 2,12 & 40 & 3 & 71 & 74 & 4900 \\
\hline Qatar & 11 & 2,3 & 3,0 & 11 & 1 & 1,0 & 2 & 7 & 2,1 & 2,07 & 14 & 1 & 78 & 79 & 123860 \\
\hline Syrie & 185 & 22,0 & 36,7 & 24 & 4 & 2,1 & -11 & 17 & 3,0 & 2,11 & 35 & 4 & 72 & 78 & - \\
\hline Turquie & 784 & 77,2 & 93,3 & 16 & 5 & 1,1 & 0 & 10 & 2,1 & 2,11 & 25 & 8 & 71 & 78 & 18760 \\
\hline Yémen & 528 & 26,0 & 38,8 & 36 & 8 & 2,8 & -1 & 68 & 4,4 & 2,33 & 42 & 3 & 62 & 64 & 3820 \\
\hline Asie centre-sud & 10790 & 1874 & 2516 & 22 & 7 & 1,6 & -1 & 47 & 2,5 & 2,27 & 31 & 5 & 65 & 68 & 5600 \\
\hline Afghanistan & 652 & 31,3 & 56,5 & 35 & 8 & 2,7 & -3 & 74 & 5,1 & 2,40 & 46 & 2 & 59 & 62 & 2000 \\
\hline Bangladesh & 144 & 158,5 & 201,9 & 20 & 6 & 1,5 & -3 & 33 & 2,2 & 2,18 & 29 & 5 & 70 & 71 & 2810 \\
\hline Bhoutan & 47 & 0,7 & 1,1 & 22 & 6 & 1,5 & 0 & 47 & 2,5 & 2,22 & 30 & 5 & 67 & 68 & 7210 \\
\hline Inde & 3287 & 1296 & 1657 & 22 & 7 & 1,5 & 0 & 44 & 2,4 & 2,30 & 31 & 5 & 65 & 68 & 5350 \\
\hline Iran & 1648 & 77,4 & 99,2 & 19 & 5 & 1,4 & 0 & 16 & 1,8 & 2,12 & 24 & 5 & 72 & 76 & 15600 \\
\hline Kazakstan & 2725 & 17,3 & 24,5 & 23 & 8 & 1,5 & 0 & 28 & 2,7 & 2,16 & 25 & 7 & 65 & 74 & 20570 \\
\hline Kirghizstan & 200 & 5,8 & 9,4 & 28 & 7 & 2,1 & -1 & 27 & 3,2 & 2,16 & 31 & 4 & 66 & 74 & 3070 \\
\hline Maldives & 0,3 & 0,4 & 0,5 & 23 & 3 & 1,9 & 0 & 9 & 2,3 & 2,10 & 26 & 5 & 73 & 75 & 9890 \\
\hline Népal & 147 & 27,1 & 35,2 & 22 & 7 & 1,5 & -3 & 46 & 2,4 & 2,20 & 34 & 5 & 67 & 69 & 2260 \\
\hline Ouzbékistan & 447 & 30,7 & 38,1 & 22 & 5 & 1,8 & -1 & 44 & 2,4 & 2,19 & 28 & 4 & 65 & 72 & 5340 \\
\hline Pakistan & 796 & 194,0 & 348,0 & 28 & 8 & 2,0 & -3 & 74 & 3,8 & 2,31 & 38 & 4 & 63 & 66 & 4920 \\
\hline Sri Lanka & 66 & 20,7 & 23,0 & 18 & 6 & 1,2 & -3 & 9 & 2,1 & 2,09 & 26 & 8 & 71 & 77 & 9470 \\
\hline Tadjikistan & 143 & 8,3 & 14,8 & 34 & 7 & 2,7 & -5 & 57 & 3,8 & 2,24 & 36 & 3 & 64 & 71 & 2500 \\
\hline Turkménistan & 488 & 5,3 & 6,6 & 22 & 8 & 1,4 & -1 & 47 & 2,4 & 2,20 & 28 & 4 & 61 & 70 & 12920 \\
\hline Asie du Sud-Est & 4497 & 621 & 831 & 19 & 7 & 1,3 & 0 & 28 & 2,4 & 2,16 & 27 & 6 & 68 & 73 & 9130 \\
\hline Birmanie-Myanmar & 677 & 53,7 & 58,6 & 17 & 9 & 0,9 & -1 & 49 & 2,0 & 2,23 & 25 & 5 & 63 & 67 & - \\
\hline Brunei & 6 & 0,4 & 0,5 & 16 & 3 & 1,3 & 1 & 4 & 1,6 & 2,07 & 25 & 5 & 77 & 80 & 68090 \\
\hline Cambodge & 181 & 14,8 & 21,7 & 24 & 6 & 1,8 & -2 & 45 & 2,8 & 2,22 & 31 & 6 & 61 & 66 & 2890 \\
\hline Indonesie & 1905 & 251,5 & 365,3 & 20 & 6 & 1,4 & -1 & 32 & 2,6 & 2,14 & 29 & 5 & 69 & 73 & 9260 \\
\hline Laos & 237 & 6,8 & 9,1 & 26 & 6 & 2,0 & -2 & 68 & 3,2 & 2,19 & 35 & 4 & 66 & 69 & 4570 \\
\hline Malaisie & 330 & 30,1 & 41,1 & 17 & 5 & 1,3 & 3 & 7 & 2,1 & 2,09 & 26 & 6 & 73 & 77 & 22460 \\
\hline Philippines & 300 & 100,1 & 157,1 & 24 & 6 & 1,8 & -1 & 23 & 3,0 & 2,14 & 34 & 4 & 65 & 72 & 7820 \\
\hline Singapour & 0,7 & 5,5 & 7,0 & 9 & 5 & 0,5 & 12 & 2 & 1,2 & 2,09 & 16 & 11 & 80 & 85 & 76850 \\
\hline Thaillande & 513 & 66,4 & 61,0 & 12 & 8 & 0,4 & 0 & 11 & 1,8 & 2,11 & 18 & 10 & 71 & 78 & 13510 \\
\hline Timor oriental & 15 & 1,2 & 2,9 & 37 & 10 & 2,7 & 0 & 63 & 5,7 & 2,22 & 42 & 5 & 61 & 62 & 6410 \\
\hline Viêt Nam & 332 & 90,7 & 106,3 & 17 & 7 & 1,0 & 0 & 15 & 2,1 & 2,16 & 24 & 7 & 70 & 76 & 5030 \\
\hline Asie rrienta & 1761 & 6010 & 15190 & 12 & 7 & 0 & 0 & 11 & 15 & 223 & 16 & 11 & 75 & 78 & 14440 \\
\hline Chine & 9561 & 1364,1 & 1311,8 & 12 & 7 & 0,5 & 0 & 14 & $\begin{array}{l}1,5 \\
1,6\end{array}$ & 2,23 & 16 & 10 & 74 & 77 & 11850 \\
\hline Corée du Nord & 121 & 24,9 & 27,0 & 15 & 9 & 0,5 & 0 & 26 & 2,0 & 2,15 & 22 & 9 & 65 & 73 & - \\
\hline Corée du Sud & 100 & 50,4 & 48,1 & 9 & 5 & 0,3 & 0 & 2,9 & 1,2 & 2,09 & 15 & 12 & 78 & 85 & 33440 \\
\hline Hong Kong - Chine & 1,1 & 7,2 & 8,6 & 8 & 6 & 0,2 & 4 & 1,6 & 1,1 & 2,08 & 11 & 15 & 81 & 87 & 54260 \\
\hline Japon & 378 & 127,1 & 97,1 & 8 & 10 & $-0,2$ & 1 & 1,9 & 1,4 & 2,07 & 13 & 26 & 80 & 86 & 37630 \\
\hline Macao - Chine & 0,03 & 0,6 & 0,8 & 11 & 3 & 0,8 & 35 & 3 & 1,2 & 2,07 & 11 & 8 & 79 & 86 & 112180 \\
\hline Mongolie & 1564 & 2,9 & 4,3 & 28 & 6 & 2,3 & 0 & 26 & 2,9 & 2,11 & 28 & 4 & 64 & 72 & 8810 \\
\hline Taïwan & 36 & 23,4 & 21,0 & 8 & 7 & 0,1 & 1 & 3,7 & 1,1 & 2,12 & 14 & 12 & 76 & 83 & - \\
\hline
\end{tabular}


DHES POPUTLANHONS

\begin{tabular}{|c|c|c|c|c|c|c|c|c|c|c|c|c|c|c|c|}
\hline & \multirow{2}{*}{$\begin{array}{c}\text { Superficie } \\
\text { (milliers } \\
\text { de } \mathrm{km}^{2} \text { ) }\end{array}$} & \multirow{2}{*}{$\begin{array}{c}\text { Population } \\
\text { mi-2014 } \\
\text { (millions) }\end{array}$} & \multirow{2}{*}{$\begin{array}{l}\text { Projection } \\
\text { popula- } \\
\text { tion } 2050 \\
\text { (millions) }\end{array}$} & \multirow{2}{*}{$\begin{array}{c}\text { Taux de } \\
\text { natalité } \\
\% \text { o }\end{array}$} & \multirow{2}{*}{$\begin{array}{c}\text { Taux de } \\
\text { mortalité } \\
\% 0\end{array}$} & \multirow{2}{*}{$\begin{array}{c}\text { Accrois- } \\
\text { sement } \\
\text { naturel } \%\end{array}$} & $\begin{array}{l}\text { Accrois- } \\
\text { sement }\end{array}$ & $\begin{array}{l}\text { Taux de } \\
\text { mortalité }\end{array}$ & $\begin{array}{l}\text { Indica- } \\
\text { teur de }\end{array}$ & Seuil de & $\begin{array}{c}\% \\
\text { moins }\end{array}$ & $\% 65$ & $\begin{array}{r}\text { Espé } \\
\text { de }\end{array}$ & & $\begin{array}{c}\text { PNB/h } \\
\text { PPA }\end{array}$ \\
\hline & & & & & & & $\underset{\%}{\text { migratoire }}$ & $\begin{array}{c}\text { infantile } \\
\% 0\end{array}$ & $\begin{array}{l}\text { fécon- } \\
\text { dité }\end{array}$ & $\begin{array}{l}\text { rempla- } \\
\text { cement }\end{array}$ & $\begin{array}{c}\text { de } \\
15 \text { ans }\end{array}$ & ou + & H & $\mathbf{F}$ & $\begin{array}{l}\text { (US \$) } \\
2013\end{array}$ \\
\hline Europe & 23061 & 741 & 726 & 11 & 11 & 0,0 & 2 & 6 & 1,6 & 2,09 & 16 & 17 & 74 & 81 & 30010 \\
\hline Union Européenne & 4323 & 507 & 514 & 10 & 10 & 0,0 & 1 & 4 & 1,5 & 2,08 & 16 & 18 & 77 & 83 & 34220 \\
\hline Europe septentrionale & 1807 & 102 & 119 & 12 & 9 & 0,3 & 2 & 3 & 1,8 & 2,07 & 18 & 17 & 78 & 83 & 37860 \\
\hline Danemark & 43 & 5,6 & 6,1 & 10 & 9 & 1,0 & 5 & 3 & 1,7 & 2,07 & 17 & 18 & 78 & 82 & 44440 \\
\hline Estonie & 45 & 1,3 & 1,2 & 11 & 12 & $-1,0$ & -5 & 2,1 & 1,5 & 2,09 & 16 & 18 & 71 & 81 & 24230 \\
\hline Finlande & 338 & 5,5 & 6,1 & 11 & 9 & 1,0 & 3 & 1,8 & 1,8 & 2,06 & 16 & 19 & 78 & 84 & 38480 \\
\hline Irlande & 70 & 4,6 & 5,7 & 15 & 7 & 0,9 & -7 & 3,3 & 2,0 & 2,09 & 22 & 12 & 78 & 83 & 35090 \\
\hline Islande & 103 & 0,3 & 0,4 & 13 & 7 & 0,7 & 5 & 1,8 & 1,9 & 2,07 & 21 & 13 & 81 & 84 & 38870 \\
\hline Lettonie & 65 & 2,0 & 1,4 & 10 & 14 & $-0,4$ & -12 & 3,6 & 1,5 & 2,09 & 14 & 19 & 69 & 79 & 21390 \\
\hline Lituanie & 65 & 2,9 & 2,5 & 10 & 14 & $-0,4$ & -6 & 3,6 & 1,6 & 2,08 & 15 & 18 & 68 & 79 & 23080 \\
\hline Norvège & 385 & 5,1 & 6,7 & 12 & 8 & 0,4 & 8 & 2,5 & 1,8 & 2,07 & 18 & 16 & 80 & 84 & 66520 \\
\hline Royaume-Uni & 243 & 64,5 & 77,0 & 12 & 9 & 0,3 & 3 & 3,9 & 1,9 & 2,07 & 18 & 17 & 79 & 83 & 35760 \\
\hline Suède & 450 & 9,7 & 11,4 & 12 & 9 & 0,2 & 2 & 2,3 & 1,9 & 2,07 & 17 & 19 & 80 & 84 & 44660 \\
\hline Europe occidentale & 1110 & 190 & 198 & 10 & 10 & 0,0 & 3 & 3 & 1,7 & 2,08 & 16 & 19 & 78 & 84 & 42220 \\
\hline Allemagne & 357 & 80,9 & 76,2 & 8 & 11 & $-0,2$ & 5 & 3,3 & 1,4 & 2,07 & 13 & 21 & 78 & 83 & 44540 \\
\hline Autriche & 84 & 8,5 & 9,4 & 9 & 9 & 0,0 & 6 & 3,1 & 1,4 & 2,07 & 14 & 18 & 79 & 83 & 43810 \\
\hline Belgique & 31 & 11,2 & 12,6 & 11 & 10 & 0,2 & 4 & 3,8 & 1,8 & 2,07 & 17 & 18 & 78 & 83 & 40280 \\
\hline France & 552 & 64,1 & 72,1 & 12 & 9 & 0,3 & 1 & 3,6 & 2,0 & 2,07 & 18 & 18 & 79 & 85 & 37580 \\
\hline Liechtenstein & 0,2 & 0,0 & 0,04 & 10 & 6 & 0,4 & 4 & 3,3 & 1,5 & - & 16 & 15 & 79 & 84 & - \\
\hline Luxembourg & 3 & 0,6 & 0,7 & 11 & 7 & 0,4 & 19 & 3,5 & 1,6 & 2,06 & 17 & 14 & 80 & 84 & 59750 \\
\hline Monaco & 0,001 & 0,0 & 0,05 & 6 & 7 & $-0,1$ & 23 & - & 1,4 & - & 13 & 24 & - & - & - \\
\hline Pays-Bas & 42 & 16,9 & 17,9 & 10 & 8 & 0,2 & 1 & 3,8 & 1,7 & 2,07 & 17 & 17 & 79 & 83 & 43210 \\
\hline Suisse & 41 & 8,2 & 9,0 & 10 & 8 & 0,2 & 6 & 3,6 & 1,5 & 2,07 & 15 & 18 & 81 & 85 & 53920 \\
\hline Europe orientale & 18827 & 294 & 262 & 12 & 13 & $-0,1$ & 1 & 8 & 1,5 & 2,10 & 15 & 14 & 68 & 77 & 19930 \\
\hline Biélorussie & 208 & 9,5 & 9,0 & 13 & 13 & $-0,1$ & 1 & 4,3 & 1,7 & 2,09 & 15 & 14 & 67 & 78 & 16940 \\
\hline Bulgarie & 111 & 7,2 & 5,7 & 9 & 14 & $-0,5$ & 0 & 7,3 & 1,5 & 2,10 & 14 & 20 & 71 & 78 & 15200 \\
\hline Hongrie & 93 & 9,9 & 9,4 & 9 & 13 & $-0,4$ & 1 & 5,1 & 1,3 & 2,08 & 14 & 17 & 71 & 78 & 20930 \\
\hline Moldavie & 34 & 4,1 & 2,1 & 11 & 11 & 0,0 & 0 & 14 & 1,2 & 2,11 & 16 & 10 & 67 & 75 & 5190 \\
\hline Pologne & 313 & 38,5 & 34,8 & 10 & 10 & $-0,1$ & 0 & 4,6 & 1,2 & 2,08 & 15 & 14 & 73 & 81 & 22300 \\
\hline République Tchèque & 79 & 10,5 & 11,1 & 10 & 10 & 0,0 & 0 & 2,5 & 1,5 & 2,07 & 15 & 17 & 75 & 81 & 25530 \\
\hline Roumanie & 238 & 20,0 & 16,5 & 10 & 12 & $-0,3$ & 0 & 8,5 & 1,3 & 2,10 & 16 & 16 & 70 & 78 & 18060 \\
\hline Russie & 17098 & 143,7 & 134,1 & 13 & 13 & 0,0 & 2 & 10,3 & 1,7 & 2,11 & 16 & 13 & 65 & 76 & 23200 \\
\hline Slovaquie & 49 & 5,4 & 5,0 & 10 & 10 & 0,1 & 0 & 5,5 & 1,3 & 2,07 & 15 & 14 & 72 & 79 & 24930 \\
\hline Ukraine & 604 & 42,9 & 33,8 & 11 & 15 & $-0,4$ & 1 & 7 & 1,5 & 2,11 & 15 & 15 & 66 & 76 & 8960 \\
\hline Europe méridionale & 1317 & 154 & 147 & 9 & 10 & $-0,1$ & -1 & 4 & 1,3 & 2,11 & 15 & 19 & 78 & 83 & 28960 \\
\hline Albanie & 29 & 3,0 & 2,9 & 12 & 7 & 0,5 & -6 & 14,4 & 1,8 & 2,12 & 19 & 12 & 75 & 80 & 10520 \\
\hline Andorre & 0,5 & 0,1 & 0,1 & 8 & 3 & 0,5 & -7 & 3,4 & 1,1 & - & 15 & 13 & - & - & - \\
\hline Bosnie-Herzégovine & 51 & 3,8 & 3,3 & 8 & 9 & $-0,1$ & 0 & 5 & 1,3 & 2,10 & 16 & 16 & 74 & 79 & 9820 \\
\hline Croatie & 57 & 4,2 & 3,6 & 10 & 13 & $-0,3$ & -1 & 3,6 & 1,6 & 2,08 & 15 & 19 & 74 & 80 & 20370 \\
\hline Espagne & 506 & 46,5 & 42,3 & 9 & 8 & 0,1 & -4 & 3,1 & 1,3 & 2,08 & 15 & 18 & 79 & 85 & 31850 \\
\hline Grèce & 132 & 11,0 & 9,4 & 9 & 11 & $-0,1$ & -4 & 2,9 & 1,3 & 2,08 & 15 & 20 & 78 & 83 & 25630 \\
\hline Italie & 301 & 61,3 & 63,5 & 9 & 10 & $-0,1$ & 3 & 3,2 & 1,4 & 2,08 & 14 & 21 & 80 & 84 & 34100 \\
\hline Kosovo & 11 & 1,8 & 1,9 & 15 & 4 & 1,1 & -2 & 11 & 2,0 & - & 28 & 7 & 67 & 71 & 8940 \\
\hline Macédoine & 26 & 2,1 & 1,8 & 11 & 9 & 0,2 & 1 & 10 & 1,5 & 2,08 & 17 & 12 & 73 & 77 & 11520 \\
\hline Malte & 0,3 & 0,4 & 0,4 & 10 & 8 & 0,2 & 7 & 5,7 & 1,4 & 2,08 & 15 & 16 & 78 & 82 & 26400 \\
\hline Monténégro & 14 & 0,6 & 0,8 & 12 & 10 & 0,3 & -3 & 5,2 & 1,7 & 2,10 & 19 & 13 & 72 & 77 & 14600 \\
\hline Portugal & 92 & 10,4 & 9,1 & 8 & 10 & $-0,2$ & -4 & 3 & 1,2 & 2,08 & 15 & 19 & 77 & 83 & 25350 \\
\hline Saint Marin & 0,06 & 0,0 & 0,04 & 10 & 7 & 0,3 & 5 & 6,4 & 1,5 & - & 15 & 18 & 82 & 86 & - \\
\hline Serbie & 88 & 7,1 & 6,4 & 9 & 14 & $-0,5$ & 0 & 5,4 & 1,4 & 2,08 & 14 & 18 & 72 & 77 & 12020 \\
\hline Slovénie & 20 & 2,1 & 2,1 & 10 & 9 & 0,1 & 0 & 2,9 & 1,5 & 2,07 & 15 & 17 & 77 & 83 & 27680 \\
\hline Océanie & 8563 & 39,0 & 60 & 18 & 7 & 1,1 & 6 & 21 & 2,4 & 2,18 & 24 & 11 & 75 & 79 & 30100 \\
\hline Australie & 7741 & 23,5 & 36,4 & 13 & 6 & 0,7 & 10 & 3,3 & 1,9 & 2,07 & 19 & 14 & 80 & 84 & 42540 \\
\hline Micronésie & 0,7 & 0,1 & 0,1 & 24 & 5 & 1,9 & -19 & 29 & 3,5 & 2,14 & 34 & 4 & 69 & 72 & 3840 \\
\hline Fidji & 18 & 0,9 & 1,0 & 21 & 8 & 1,4 & -6 & 15 & 2,5 & 2,13 & 29 & 5 & 67 & 73 & 7610 \\
\hline Guam & 0,5 & 0,2 & 0,3 & 22 & 5 & 1,7 & -6 & 13,4 & 3,1 & 2,09 & 27 & 7 & 75 & 81 & - \\
\hline Iles Marshall & 0,2 & 0,1 & 0,1 & 30 & 4 & 2,5 & -17 & 26 & 4,1 & - & 40 & 2 & 67 & 73 & 4620 \\
\hline Iles Salomon & 29 & 0,6 & 0,4 & 31 & 5 & 2,6 & 0 & 26 & 4,1 & 2,23 & 39 & 3 & 67 & 74 & 1810 \\
\hline Kiribati & 0,7 & 0,1 & 0,2 & 30 & 9 & 2,1 & -1 & 45 & 3,8 & 2,19 & 35 & 4 & 58 & 66 & 2780 \\
\hline Nauru & 0,02 & 0,0 & 0,1 & 36 & 8 & 2,9 & -9 & 39 & 4,3 & - & 37 & 1 & 58 & 63 & - \\
\hline Nouvelle-Calédonie & 19 & 0,3 & 0,3 & 17 & 5 & 1,2 & 4 & 5 & 2,2 & 2,19 & 24 & 8 & 74 & 81 & - \\
\hline Nouvelle-Zélande & 271 & 4,3 & 5,5 & 13 & 7 & 0,7 & 5 & 4,4 & 2,0 & 2,08 & 20 & 14 & 80 & 83 & 30750 \\
\hline Palau & 0,5 & 0,0 & 0,1 & 14 & 9 & 0,5 & 0 & 11 & 1,7 & - & 20 & 6 & 69 & 75 & 14540 \\
\hline Papouasie-Nouv. Guinée & 463 & 7,6 & 14,2 & 33 & 10 & 2,3 & 0 & 48 & 4,3 & 2,31 & 39 & 3 & 60 & 65 & 2430 \\
\hline Polynésie française & 4 & 0,3 & 0,3 & 17 & 6 & 1,1 & -6 & 5,1 & 2,1 & 2,08 & 25 & 6 & 73 & 78 & - \\
\hline Samoa occidentale & 3 & 0,2 & 0,2 & 29 & 5 & 2,4 & -24 & 16 & 4,7 & 2,15 & 39 & 5 & 73 & 76 & 4840 \\
\hline Tonga & 0,7 & 0,1 & 0,1 & 27 & 7 & 2,0 & -19 & 17 & 3,9 & 2,13 & 37 & 6 & 69 & 73 & 5450 \\
\hline Tuvalu & 0,03 & 0,0 & 0,1 & 25 & 9 & 1,6 & 0 & 10 & 3,2 & - & 33 & 5 & 67 & 72 & 5990 \\
\hline Vanuatu & 12 & 0,3 & 0,5 & 29 & 5 & 2,4 & 0 & 21 & 4,4 & 2,15 & 39 & 4 & 70 & 73 & 2840 \\
\hline
\end{tabular}




\section{Estimations ou résultats ?}

La valeur des données est indicative. Il s'agit d'estimations pour l'année 2014, faites parfois plusieurs années avant que les statistiques ne soient enfin disponibles. En outre, dans un certain nombre de pays, la médiocre qualité de l'état civil contraint à recourir à des estimations fondées sur des données ou des modèles démographiques fragmentaires. La qualité des données peut varier considérablement même à l'intérieur d'un État. C'est dans le domaine des migrations internationales que l'imprécision est la plus grande, ce qui, dans la mesure où elles interviennent dans le calcul de certains indicateurs, peut aggraver le degré d'incertitude des estimations ${ }^{1}$.

Les variations brusques de certains indices (fécondité, espérance de vie, mortalité infantile ou solde migratoire) depuis la livraison précédente ${ }^{2}$ résultent souvent plus d'un ajustement des estimations que de changements réels.

1. Le signe « - » signifie que certaines données manquent.

2. Sardon, Jean-Paul, « La population des continents et des États en 2013 », Population \& Avenir, $n^{\circ}$ 715, novembre-décembre 2013.

\section{L'Afrique, quart de l'humanité en 2050 ?}

Selon les projections, l'Afrique serait le seul continent à voir sa part dans la population mondiale progresser notablement, en dépit de sa forte mortalité, du fait de sa fécondité élevée et de la jeunesse de sa population, qui confère à sa croissance une grande inertie. Sa part pourrait passer de $15,7 \%$ à $25,1 \%$ en 2050. Celle de l'Amérique pourrait se tasser légèrement (de $13,4 \%$ à $12,6 \%$ ) ; celle de l'Asie baisser un peu (de 60,1\% à $54,2 \%)$; mais celle de l'Europe reculer de $10,2 \%$ à 7,5\% ${ }^{6}$.

L'Inde serait alors le pays le plus peuplé avec près de 1,7 milliard d'habitants, soit 350 millions de plus quen Chine. Le Nigeria et les États-Unis suivraient avec près de 400 millions.

La population de l'Afrique serait multipliée par 2,1 d'ici 2050 et même par 2,6 en Afrique centrale. En 2050, l'Afrique pourrait compter 6 pays de plus 100 millions d'habitants, dont le Nigeria (396,5 millions) et la République démocratique du Congo (193,6 millions) qui se situeraient au $3^{e}$ et au $9^{e}$ rang mondial, auxquels s'ajouteraient l'Éthiopie, l'Égypte, la Tanzanie et l'Ouganda.

6. Dumont, Gérard-François, « Afrique-Europe : l'inversion démographique », dans : Cluzel, Jean, Solidarité Europe-Afrique, Paris, Economica, 2013.
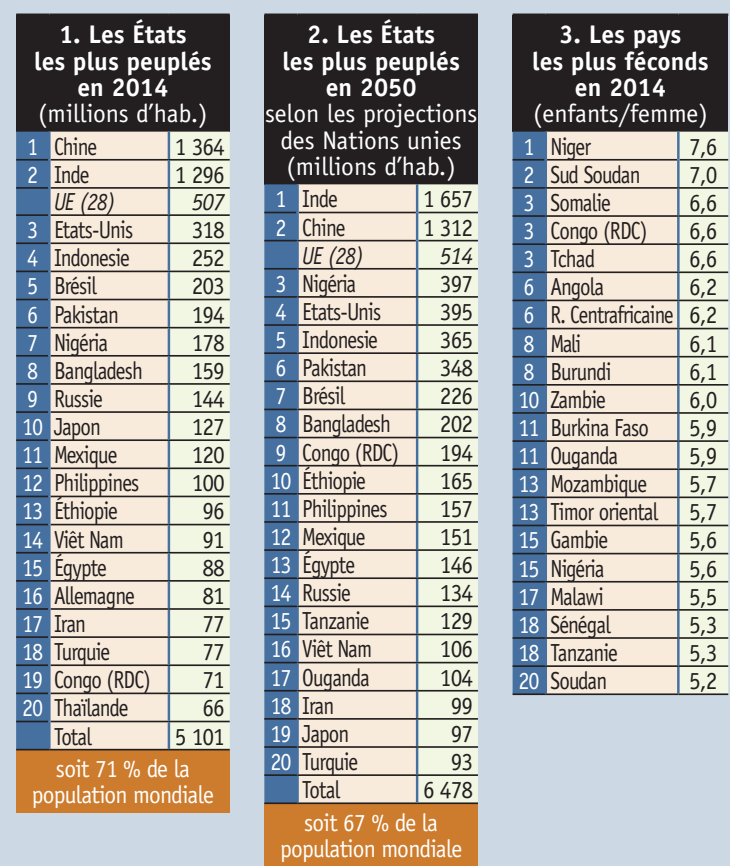

4. Les champions de la croissance naturelle en 2014 (taux annuel \%.)

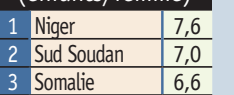

3 Somalie

\begin{tabular}{|l|l}
\hline Congo (RDC) & 6,6 \\
\hline
\end{tabular}

3 Tchad

\begin{tabular}{ll|l|}
\hline 6 & Angola & 6,2 \\
\hline 6 & R. Centrafricaine & 6,2 \\
\hline
\end{tabular}

\begin{tabular}{lll}
\hline 8 & Mali & 6,1 \\
\hline
\end{tabular}

8 Burundi

10 Zambie

11 Burkina Faso $\frac{6,0}{5,9}$

11 Ouganda

13 Mozambique 5,7

13 Timor oriental 5,7

15 Gambie

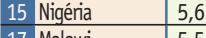

18 Sénégal

\begin{tabular}{ll|l}
18 & Tanzanie & 5,3 \\
\hline 20 & Soudan & 5,2 \\
\hline
\end{tabular}

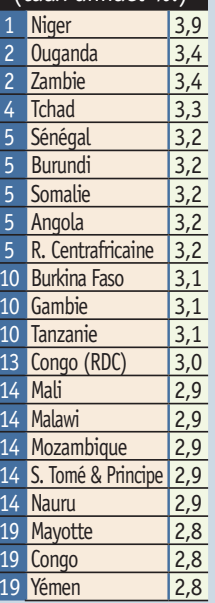

\section{Les tristes ecords de mortalite infantile en 2014}

(quotients pour

1 R. Centrafricaine 116 2 Congo (RDC) 109

3 Tchad

\begin{tabular}{ll|l}
3 & Tchad & 96 \\
\hline 5 & Guinée-Bissau & 94 \\
\hline & &
\end{tabular}

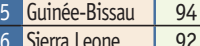

6 Sierra Leone $\quad 92$

\begin{tabular}{lll|l}
7 & Guinee équator. & 89 \\
\hline 8 & Burundi & 87 \\
\hline & Mozanbique & 85 \\
\hline
\end{tabular}

\begin{tabular}{cl|r}
9 & Mozambique & 85 \\
\hline 10 & Lesotho & 82 \\
\hline
\end{tabular}

10 Lesotho

11 Somalie

12 Sud Soudan

13 Côte d'Ivoire

14 Afghanistan

14 Pakistan

16 Mauritanie

17 Burkina Faso

18 Bénin

18 Nigéria

20 Yémen

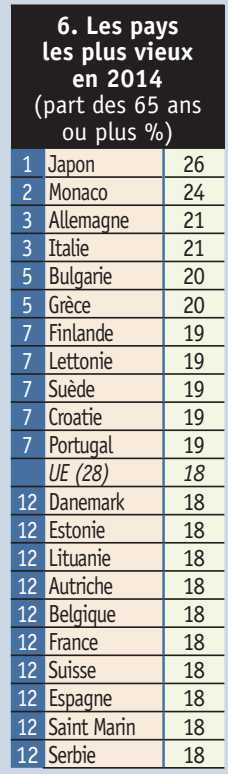

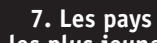

les plus jeunes en 2014

part des moins

de 15 ans \%)

\begin{tabular}{ll|l}
1 & Niger & 50 \\
\hline 2 & Tchad & 49 \\
\hline 3 & Mad & 48 \\
\hline
\end{tabular}

\begin{tabular}{lll|l}
3 & Mali & 48 \\
\hline 3 & Ouganda & 48 \\
\hline & Somale &
\end{tabular}

\begin{tabular}{ll|l}
3 & Angola & 48 \\
\hline 7 & &
\end{tabular}

\begin{tabular}{l|l|l}
7 & Burkina Faso & 46 \\
\hline 8 & Gambie & 46 \\
\hline
\end{tabular}

\begin{tabular}{ll|l}
8 & Gambie & 46 \\
\hline 8 & Congo (RDC) & 46 \\
\hline
\end{tabular}

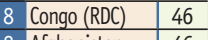

8 Afghanis

12 Burundi

\begin{tabular}{ll}
12 Malawi & 45 \\
\hline
\end{tabular}

\begin{tabular}{ll|l}
12 & Mayotte & 45 \\
\hline 12
\end{tabular}

12 Mozambique 45

Tanzanie

17 Nigéria

17 Sénég

19 Bénin

19 Libéria

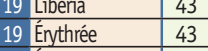

19 Éthiopie 43 \begin{tabular}{lll}
3 & Ouganda & 48 \\
\hline 3 & Somalie & 48 \\
\hline
\end{tabular}

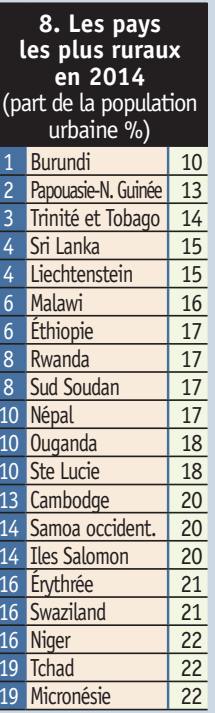

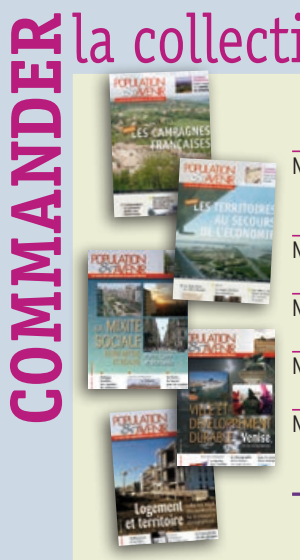

\section{tion 2013 de POPULATION \& AVENIR}

\begin{tabular}{|c|c|c|c|c|}
\hline \multirow{2}{*}{\multicolumn{2}{|c|}{ La collection 2013 complète (nº 711 à 715) }} & \multicolumn{2}{|c|}{\begin{tabular}{l|l} 
Prix & Nb ex.
\end{tabular}} & \multirow[b]{2}{*}{.......... $€$} \\
\hline & & $40 €$ & & \\
\hline 715 & $\begin{array}{l}\text { - Les campagnes françaises: un renouveau incontestable mais très inégal. } \\
\text { - Usages et paysages d'un monde en mutation : un bourg en Bulgarie } \\
\left.\text { (dos. pédag., } 6^{e}\right) \text {. }\end{array}$ & $10 €$ & & ..........€ \\
\hline No 714 & $\begin{array}{l}\text { - Les territoires au secours de l'économie. } \\
\text { - Presse et radio dans la France occupée (dos. pédag., Term ES et L). }\end{array}$ & $10 €$ & & ..........€ \\
\hline 713 & $\begin{array}{l}\text { - La mixité sociale : entre mythe et réalité - Le cas de Paris, Lyon et Marseille. } \\
\text { - Le Sahara : ressources, conflits (dos. pédag., Term ES et L). }\end{array}$ & $10 €$ & & ..........€ \\
\hline 712 & $\begin{array}{l}\text { - Ville et développement durable : Venise, un cas exceptionnel. } \\
\text { - La Serbie, pays candidat à l'Union européenne (dos. pédag., } 3^{\mathrm{e}} \text { ). }\end{array}$ & $10 €$ & & ..........€ \\
\hline$\sqrt{ }{ }^{\circ} 711$ & $\begin{array}{l}\text { - Logement et territoire : l'effet très inégal des constructions sur } \\
\text { - la croissance démographique. } \\
\text { - Un produit dans la mondialisation : li'Phone d'Apple (dos. pédag. Term ES et L). }\end{array}$ & $10 €$ & & ..........€ \\
\hline
\end{tabular}

Règlement à adresser à :

Population \& Avenir, 35, av. Mac-Mahon, 75017 Paris

- par chèque bancaire à l'ordre de Population et Avenir

- par carte bancaire sur wumw population-

$\begin{array}{ll}\text { Nom } & \\ \text { Prénom } & \\ \text { Adresse } & \\ \text { Code postal } & \text { Ville } \\ \text { Tél. } & \text { Fax } \\ \text { Courriel }\end{array}$

